Aditi Thakur and Shaina Nagpal*

\title{
Performance Evaluation of Different Optical Amplifiers in Spectrum Sliced Free Space Optical Link
}

https://doi.org/10.1515/joc-2017-0120

Received July 16, 2017; accepted July 24, 2017

\begin{abstract}
Free space optics (FSO) is well-competent and premier technology to cater the high speed services in different geographical areas such as hilly areas and inter building network. In this paper, we successfully demonstrated the spectrum sliced wavelength division multiplexed FSO system. In order to make system bandwidth efficient, frequency spacing of $75 \mathrm{GHz}$ is taken among the wavelength division multiplexing (WDM) channels. Carrier spectrum broadening is achieved for spectrum slicing through the nonlinearity called self-phase modulation. Moreover, requirement of multiple laser sources is eliminated. However, in conventional WDM systems, $n$ numbers of lasers are needed to generate $n$ WDM channels. To strengthen the signal in this FSO system, three optical amplifiers are scrutinized such as erbium-doped fiber amplifier (EDFA), semiconductor optical amplifier (SOA) and Raman amplifier in terms of Q-factor and bit error rate (BER). Results revealed that EDFA is best amplifier in proposed SS-WDM-FSO system.
\end{abstract}

Keywords: wavelength division multiplexing (WDM), free space optics (FSO), erbium-doped fiber amplifier (EDFA), semiconductor optical amplifier (SOA), highly nonlinear fiber (HNLF)

\section{Introduction}

Optical wireless communication is the engaging technology in the field of communication and provides us with the fastest transmission than microwave and RF technology [1]. In recent times, free space optical (FSO) communication has been mostly investigated to take benefits of useful properties over optical fiber communication [2]. In

\footnotetext{
*Corresponding author: Shaina Nagpal, Department of Electronic \& Communication Engineering, Chandigarh University, Gharuan, Punjab 140413, India, E-mail: shaina.cu29@gmail.com

Aditi Thakur, Department of Electronic \& Communication Engineering, Chandigarh University, Gharuan, Punjab 140413, India, E-mail: aditiithakur89@gmail.com
}

the past, lot of researches have been carried out to cater the high speed data requirements and to propose costeffective architecture [3]. Moreover, FSO render fortify communication because of insignificant interception incorporating P2P (point to point) laser signals. Merits of FSO communications are large bandwidth and capacity, license free, operate on low power and save the cost of trenching and permit as in optical fiber communication $[4,5]$. For the geographical areas in India, where the deployment of optical fiber is not possible, FSO is a major candidate and attracted attention due to its applications and numerous advantages. Factors that affect the performance of FSO are the atmospheric degrading conditions such as mild rain, medium rain, high rain, fog and haze [6-8] that enervate the link and become the cause to shut the network transmission. To mitigate the effects of atmospheric instability, many researches have been demonstrated or proposed and numerous solutions to quell the turbulences effects [9-12]. Free space communication is evaluated in the atmospheric instability and effect of beam width also investigated in Ref. [3]. Also, orthogonal frgequency division multiplexing (OFDM) advance modulations [13], aperture averaging [14], diversity [15] and FSO signal amplification [16] are investigated for FSO channel. Wavelength division multiplexing (WDM) has been employed for FSO capacity enhancement at ultra-narrow channel spacing's and high data rates [17]. Access networks reliant on WDM support many users and are based on spectrum slices of available spectrum. Bidirectional transmission and multiplication of capacity represent the WDM as adaptive and reliable that can give wide coverage over atmospheric instabilities in FSO. Spectrum slicing is the important candidate that provides assurance for distribution of multi-wavelength intensity source in numerous end users [18]. Spectrum slicing having less complex operation as compared to WDM, which uses several intensity sources operational at different frequencies. Also, WDM systems are wavelength selective and sensitive to routing of each wavelength to specific port. So, spectrum sliced WDM has identical advantages as WDM and less complex, low cost, power efficient for future generation 
networks. Spectrum slicing is reported in researches using arrayed waveguide gratings [19], semiconductor optical amplifier (SOA) (amplifier spontaneous noise [ASE]) [20], SLDs (super-luminescent diodes) [21]. However, these systems support low data speed and number of channels, but FSO can support high speed and provide large bandwidth. An optimal method of SSWDM is needed for FSO networks.

In this research article, we accentuate on the high speed supercontinuum-based spectrum sliced WDM (SCSS-WDM) architecture. Different amplifiers such as erbium-doped fiber amplifier (EDFA), SOA and Raman amplifier are investigated over proposed architecture. Utmost approach is to cater the high speed data demands and thus system deliberated and demonstrated for $10 \mathrm{Gbps}$. High nonlinear fiber is incorporated in the system for supercontinuum generation and which provide high power broadened spectra for spectrum slicing.

\section{Self-phase modulation}

The intensity reliant traits of optical medium or fiber are prominent cause to modulation of phase as well as selffocusing cramped pulse. In optical fiber, any other confinement that is generated by intense pulse is insignificant. Nevertheless, retardation at high pulse in contrast to the trailing edge and leading will augment in extensive communication and consequences in substantial modulation of phase. Pulse enlargement is more significant when associated with high launched power ultra short pulse and link length. Since broadening of spectrum or supercontinuum generation limits the system reach; however, it is an important phenomenon to save cost by providing large spectrum from single intensity source for spectrum slicing.

Proportionality constant for highly nonlinear fiber is expressed as

$$
\gamma_{S P M}=\frac{2 \pi n^{2}}{\lambda A_{\text {eff }}}
$$

where $\gamma_{\text {SPM }}$ nonlinearity (in units of $\mathrm{rad} /(\mathrm{W} \cdot \mathrm{m})$ ) and where $A_{\text {eff }}$ is the effective area of highly nonlinear fiber. Major characteristics of refractive index change with the launched intensity of pulse.

$$
P_{n l}=\frac{3}{4} \varepsilon_{0} \chi^{3} \mid E_{0}(z, t) e^{[i(\omega t-\beta z)]}
$$

where electric field amplitude is $E_{0}(t)$, which fluctuate with time $(t)$ and frequency $(\omega)$ of launched light. Considering the effects of polarization into the wave equation, direct to refractive index change or modification over the original $n_{0}$. Where $n_{0}$ is zero field and net index is expressed as:

$$
n=n_{0}+n_{2}\left|E_{0}(z, t)\right|^{2}
$$

Nonlinearity in refractive index is shown as $n_{2}^{\prime}$

$$
n_{2}=R_{e}\left[3 \frac{\chi^{3}}{8 n_{0}}\right]
$$

The complex field travelled through the medium can be given as:

$$
E=E_{0}(z, t) e^{\left[i\left(\omega_{0} t-\left(n_{0}+n_{2} I(z, t)\right) k_{0} z\right)\right]}
$$

The instantaneous frequency is expressed as:

$$
\omega^{\prime}=\omega_{0}-n_{2} k_{0} z \frac{\partial I}{\partial t}
$$

The property and effects of SPM on pulse transmission can be qualitatively seen from eqs (4) and (5). First, extra frequency components are positioned on the pulse, thus raising its spectral width. A frequency sweep imposed on the pulse, which rely on the sign of $\partial \mathrm{I} / \partial t$. In optical fibers, latter described feature plays an important role, since the forced frequency from self-phase will accumulated or deduct from the chirp forced by linear group dispersion. The actual generation of supercontinuum in HNLF can be altered, reliant on fiber length, the time of pulse, maximum intensity and wavelength of pump. When short pulses are fed into nonlinear medium, broadening can be caused by SPM. There are many applications of supercontinuum generation, which include coherence tomography, cytometry, microscopy fluorescence, the description of optical devices and the generation of multiple carrier waves. It is significance to give emphasis on the coherence traits of supercontinua. Nevertheless, supercontinua produced by intense pulses can still have time coherence in the way that there can be sturdy correlations between the electric fields analogous to different signals, if the broadening is extremely reproducible. This type of coherence is incredibly significant for the generation of combs of frequency, and it may or may not be achieved reliant on parameters such as the seed duration of pulse, length of fiber and GVD.

\section{System setup}

In this proposed work, a communication software Optiwave Optisystem ${ }^{\mathrm{TM}}$ is used. WDM is popular technique to support high speed and high capacity systems.

However, WDM needs multiple laser sources at different wavelengths that increase the cost of the 
system. Spectrum slicing is the most attractive technique to reduce the cost of multi-wavelength architectures such as WDM. Spectrum slicing is an substitute to WDM, because it can also support data rates similar to aforementioned technique and transmit data streams in parallel tributaries. Therefore, main emphasis of this work is to generate high power and broad spectrum for slicing with the use of nonlinearity called self-phase modulation (SPM). The principle for the generation of broad spectrum is nonlinearity aroused in highly nonlinear fiber that has very low effective area $\left(10 \mu \mathrm{m}^{2}\right)$ due to coherent high pulse source. A continuous wave laser operated at $30 \mathrm{~dB}$ and $193.1 \mathrm{THz}$ fed to HNLF (highly nonlinear fiber) for supercontinuum generation as shown in Figure 1.

Figure 2 depicts the spectrum of laser signal with and without spectrum broadening. Table 1 shows the system parameters. Four frequency signals $193 \mathrm{THz}$, 193.075 THz, $193.150 \mathrm{THz}$ and $193.225 \mathrm{THz}$ are sliced with dense channel spacing of $75 \mathrm{GHz}$ to save the available bandwidth. These spectrums are modulated with non-return to zero (NRZ) linecodings and binary data is generated at $10 \mathrm{Gbps}$ with pseudo-random bit sequence generator.
Table 1: System architecture experimental parameters.

\begin{tabular}{ll}
\hline Quantity & Value \\
\hline Wavelength & $1552.52 \mathrm{~nm}$ \\
Number of channels & 4 \\
Channel spacing & $75 \mathrm{GHz}$ \\
Diameter of transmitting antenna & $5 \mathrm{~cm}$ \\
Diameter of receiving antenna & $20 \mathrm{~cm}$ \\
Beam divergence & $1 \mathrm{mrad}$ \\
Effective aperture & $10 \mu \mathrm{m}^{2}$ \\
HNLF attenuation & $0.5 \mathrm{~dB}$ \\
Nonlinear index of refraction & $2.6 \mathrm{e}-019 \mathrm{~m}^{2} / \mathrm{W}$ \\
Amplifier & EDFA, SOA, Raman \\
\hline
\end{tabular}

Mach-Zehnder modulator is biased at peak point and data is modulated with spectrum signal drive and NRZ. Signals are multiplexed as depicts in Figure 3 and amplified with different amplifiers one after another to check the performance of each amplifier. Amplifier used are EDFA, SOA and Raman amplifier and after amplification signal is fed to FSO channel that has 1 mrad beam divergence and fixed $5 \mathrm{~cm}$ transmitter aperture, $20 \mathrm{~cm}$ receiver aperture diameter.

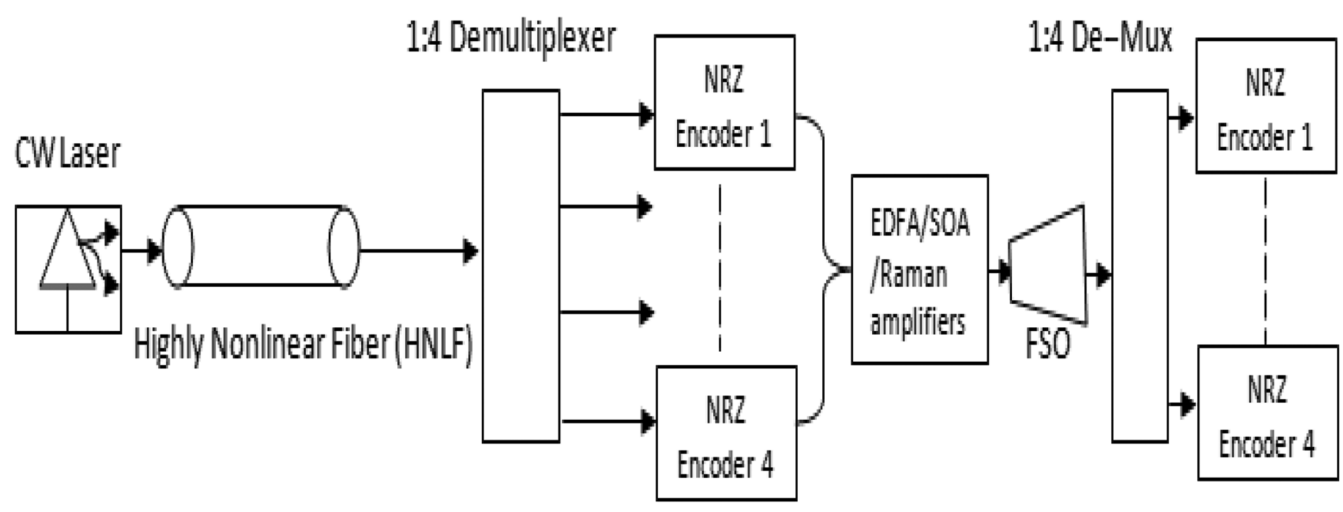

Figure 1: System architecture of supercontinuum spectrum sliced (SC-SS) WDM FSO system.
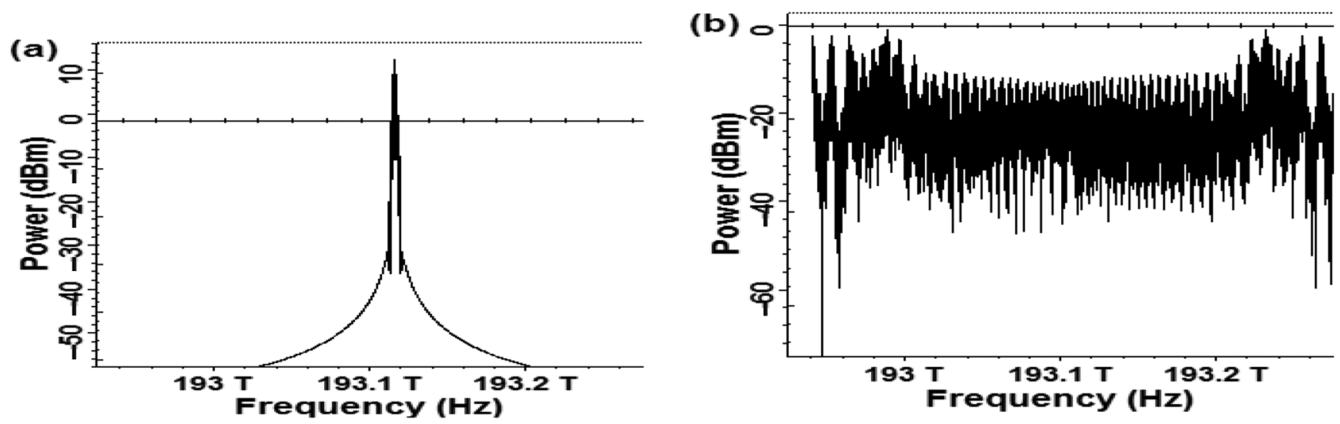

Figure 2: A continuous wave laser spectrum (a) without (b) with supercontinuum generation. 


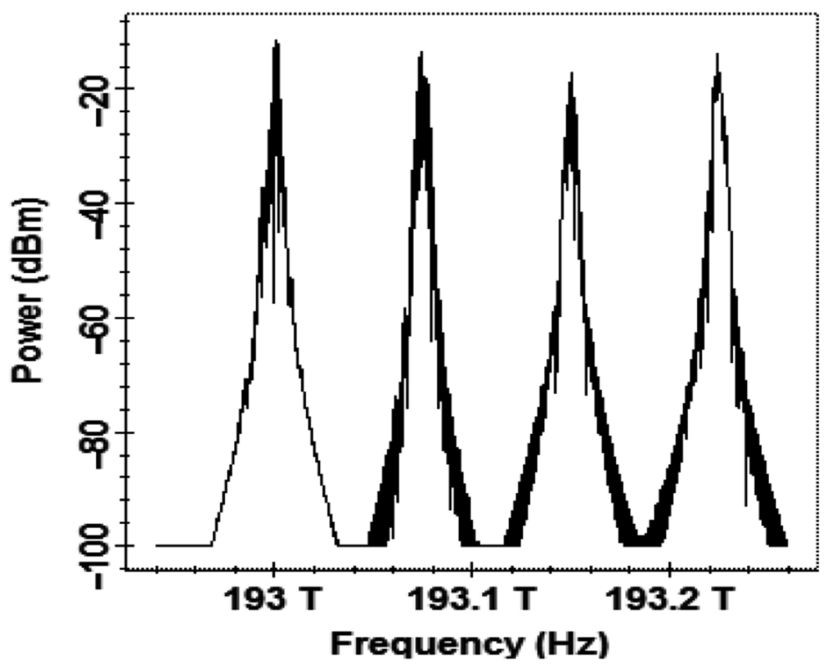

Figure 3: OSA multiplexed signals representation.

\section{Results and discussion}

FSO link length is varied from $1 \mathrm{~km}$ to $5 \mathrm{~km}$ in order to evaluate the quality of reception in different cases of different amplifiers. Maximum prolonged distance is observed in case of the EDFA amplifier and minimum results are reported for SOA. Amplification of EDFA is maximum in C-band and due to high power of input signals, SOA introduced nonlinear effects and thus degrade the performance. Graphical representation of different amplifiers is depicted in Figure 4.

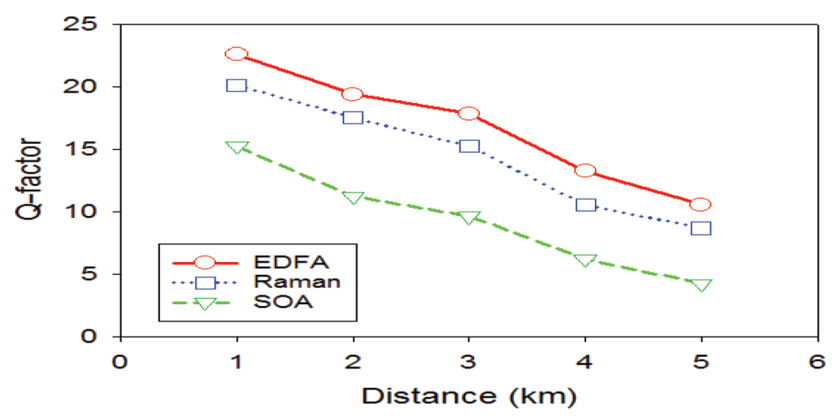

Figure 4: Graphical representations of SC-SS-WDM FSO system for different amplifiers.

It is clearly seen that Q-factor for EDFA varies from 24.32 to 10.26 for $1-5 \mathrm{~km}$ respectively. As in case of Raman and SOA is 21.86 to 8.63 and 16.54 to 4.29 respectively. Maximum range that can proposed system cover for EDFA is $>10 \mathrm{~km}$ for clear weather, $8 \mathrm{~km}$ for Raman, and $3.5 \mathrm{~km}$ for SOA. In this figure, we have shown the comparison of different amplifiers in terms of LoG (bit error rate [BER]). It is evident that BER varies inversely to Q-factor. Due to attenuation,
BER intrudes more at longer distance such as $5 \mathrm{~km}$. As shown in Figure 5, the BER of EDFA is less and tends to increase as distance increases. Performance of Raman amplifier falls just below the EDFA and SOA amplifier exhibits maximum errors. Figure 5 depicts that the performance of SC-SS-WDM in the FSO communication using EDFA is superior to Raman and SOA.

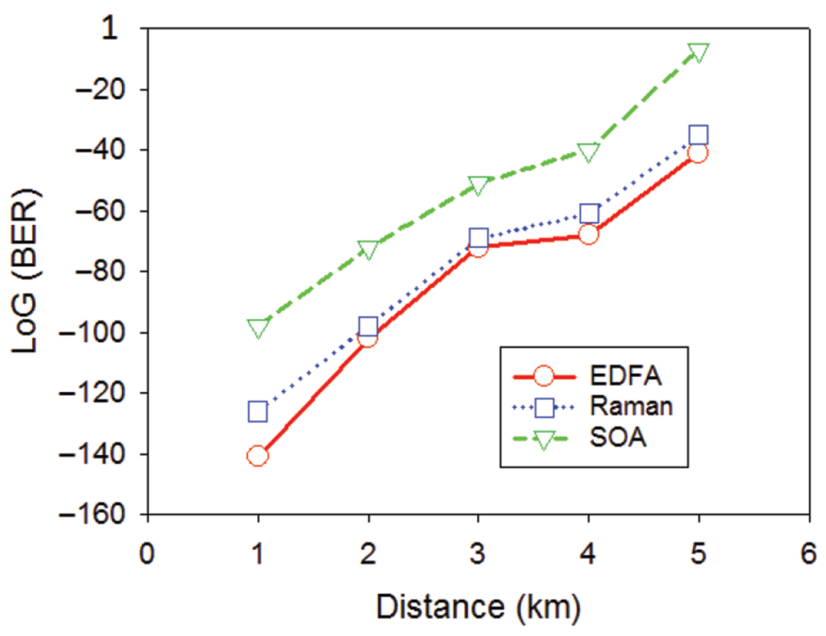

Figure 5: LoG (BER) versus distance at different distances.

Received power is investigated with the distance and it is clearly observed from Figure 6 that for lower distances, received power is more and for longer distances received power is less. More received power is seen in case of SC-SSWDM EDFA in comparison to Raman, SOA. After propagation and analysis of spectrum sliced signal, data streams are de-multiplexed respective of their wavelengths and route to respective wavelength ports. Receiver is consisting of a photodetector that convert the input light signal into electric signal. In this work, PIN with $10 \mathrm{nA}$ and $1 \mathrm{~A} / \mathrm{W}$ responsibility

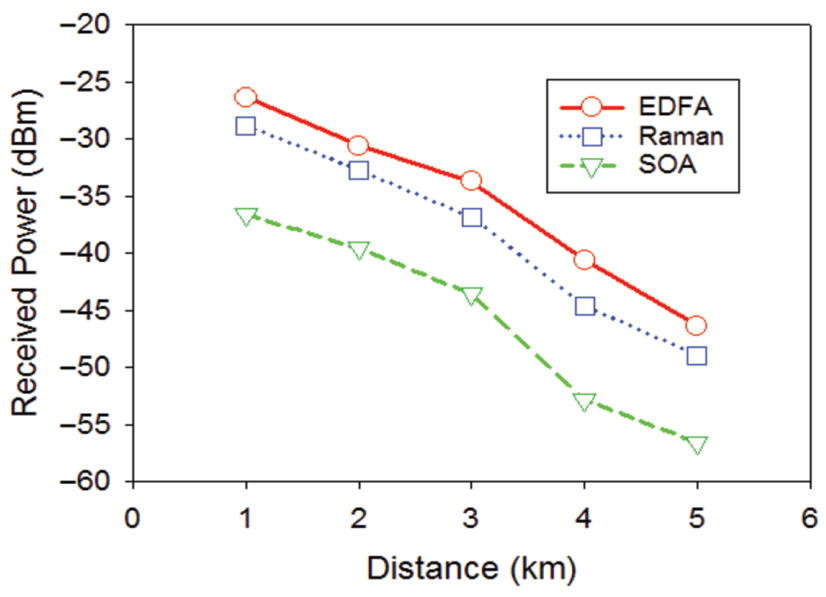

Figure 6: Received power versus distance of EDFA, Raman and SOA. 


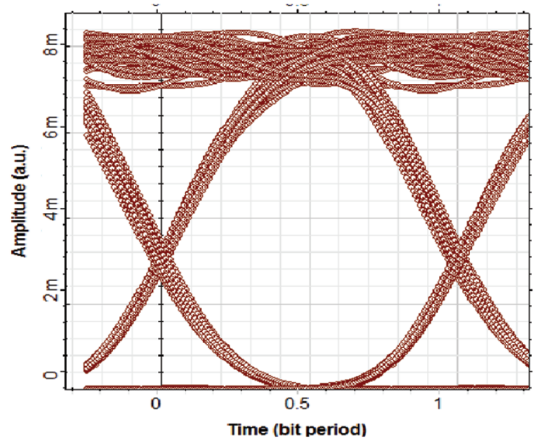

(a)

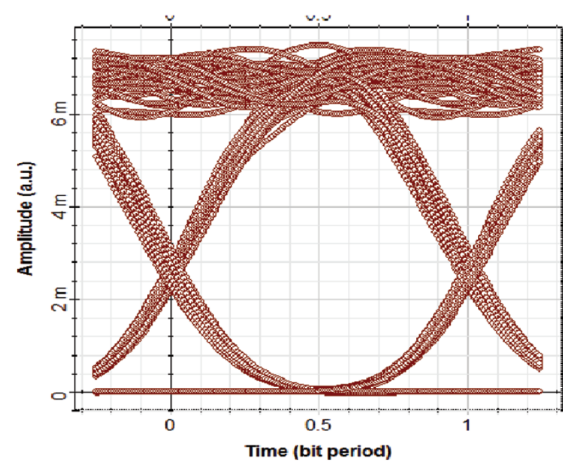

(b)

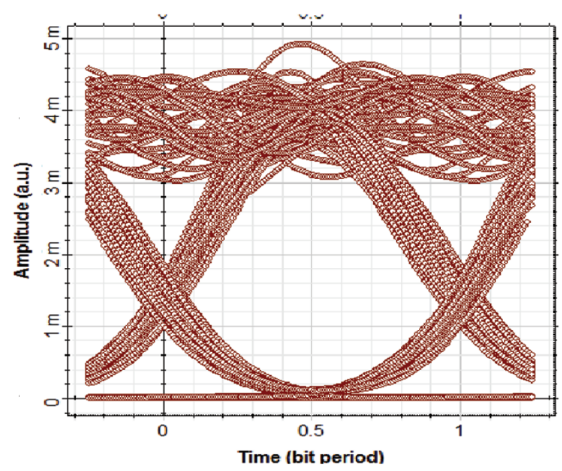

(c)

Figure 7: Eye diagram of SC-SS-WDM FSO for (a) EDFA (b) Raman (c) SOA amplifiers.

is used followed by a low-pass Bessel filter. LPF is for removing noises of the signals after optical to electric conversion done by PIN. A 3-R regenerator placed after LPF for re-timing, re-shaping and re-amplification followed by BER analyzer. BER analyzer is a decision component that show the final values of Q-factor, BER, eye closer penalty, etc. Optical spectrum analyzers (OSA) is placed to access the spectrum frequencies and power of each slices of wavelength. Optical time domain visualizers represent data bits with respective to time of each bit slot. It is observed from the Figure 7 that eye opening in SC-SS-WDM FSO using EDFA system is far better than SOA, Raman amplifiers due to better Gain of EDFA in c-band. Therefore, the demonstrated system is competent, flexible and promising for low-cost SS-WDM FSO architecture for enhanced FSO communication.

\section{Conclusion}

In this paper, a $10 \mathrm{Gbps}$ spectrum sliced dense WDM FSO communication is investigated which is based on Kerr nonlinearity supercontinuum generation. Analysis has been carried out for different amplifiers such as erbium-doped fiber amplifier, SOA and Raman amplifier. SC-SS-WDM FSO communication system exhibits better performance when EDFA is incorporated in the system. Raman amplifier performs superior to SOA in terms of Q-factor and BER. Maximum link range is observed in case of EDFA is $<10 \mathrm{~km}$ and system works for $3.5 \mathrm{~km}$ in case of SOA. Demonstrated system is promising and well competent low cost architecture for high speed FSO communication. It is perceived that BER for EDFA at $5 \mathrm{~km}$ is $10^{-33}$ and for SOA is $10^{-7}$. Results revealed that performance of SC-SS-WDM using EDFA is superior to SOA and Raman architecture.

\section{References}

1. Epple B, Simplified channel model for simulation of free- space optical communications, Journal of optical communication and networking 2010; 2(5): 293-304.

2. He J, Norwood RA, Pearce MB, Djordjevic IB, Cvjetic M, Subramaniam S, et al. A survey on recent advances in optical communications. Comput Electr Eng 2014;40:216-240.

3. Wing L, Ying F. Research on the relationship of BER of FSO system with beam width in the presence of the beam wander. J Opt Optoelectron Technol 2010;8:26-30.

4. Khalighi M, Uysal M. Survey on free space optical communication: a communication theory perspective. IEEE Commun Surveys Tutorials 2014;16:2231-2258.

5. Kazaura K, Omaea K, Suzukia T, Matsumoto M, Mutafungwa E, Asatanic $\mathrm{K}$ et al. Experimental demonstration of next-generation FSO communication system. Optics East 2006, Int'l. Society for Optics and Photonics 2006; 63 90:63900G-1 -63 900G-12.

6. Chaudhary S, Amphawan A, Nisar K. Realization of free space optics with OFDM under atmospheric turbulence. Opt Int J Light Electron Opt 2014;125:5196-5198.

7. Liu W, Shi W, Cao J, Lv Y, Yao K, Wang S, et al. Bit error rate analysis with real-time pointing errors correction in free space optical communication systems. Opt Int J Light Electron Opt 2014;125:324-328.

8. Flohberger M, Gappmair W, Koudelka W. Error performance of coded FSO links in turbulent atmosphere modeled by Gamma distribution. IEEE Trans Wireless Commun 2009;8:2209-2213.

9. Lee E, Chan V. Part 1: optical communication over the clear turbulent atmospheric channel using diversity. IEEE J Sel Areas Commun 2004;22:1896-1906.

10. Tsiftsis T, Sandalidis H, Karagiannidis G, Uysal M. Optical wireless links with spatial diversity over strong atmospheric turbulence channels. IEEE Trans Commun 2009;8:951-957.

11. Abtahi M. Suppression of turbulence-induced scintillation in free-space optical communication systems using saturated optical amplifiers. IEEE 2007;24(12):4966-497.

12. Sandalidis HG. Coded free space optical links over strong turbulence and misalignment fading channels. IEEE 2011;59:669-674. 
13. Son IK. Design and optimization of free space optical networks (PhD Thesis), Auburn University, 2010.

14. Lee IE, Ghassemlooy Z, Ng WP, Khalighi MA, Liaw SK. Effects of aperture averaging and beam width on a partially coherent Gaussian beam over free-space optical links with turbulence and pointing errors. Appl Opt 2016;55(1):1-9.

15. Abtahi M, Lemieux P, Mattlouthi W, Tusch LA. Suppression of turbulence induced scintillation in free-space optical communication systems using saturated optical amplifiers. J Lightwave Technol 2006;24:4966-4973.

16. Ciaramella E, Arimoto Y, Costestabile G, Matsumoto M. 1.28 Terabit/s $(32 \times 40 \mathrm{Gbit} / \mathrm{s})$ WDM transmission system for free space optical communications. IEEE J Sel Areas Commun 2009;27:1639-1645.

17. Tsai WS, Lu HH, Li CY, Lu TC, Lin HH, Chen BR, et al. A 50-m/ 320-Gb/s DWDM FSO communication with afocal scheme. IEEE Photonics J 2016;8.

18. Pendock GJ, Sampsom DD. Transmission performance of high bit rate spectrum sliced WDM systems. J Lightwave Technol 1996;14(10):2141-2148.
19. Rashidi F, Hea J, Chena L. Spectrum slicing WDM for FSO communication systems under the heavy rain weather. Opt Commun 2017;387:296-302.

20. Lee K, Lim SD, Jhonm YM, Kim CH, Ghelfi P, Nguyen AT, et al. Broadcasting in colorless WDM-PON using spectrumsliced wavelength conversion. Opt Fiber Technol 2012;18(2):112-116.

21. Kaneko S, Kani JI, Iwatsuki K, Ohki A, Sugo M, Kamei S. Scalability of spectrum-sliced DWDM transmission and its expansion using forward error correction. J Lightwave Technol 2006;24(3):1295-1301.

22. Fadhil AH, Amphawan A, Shamsuddin HA, Abdual TH, Al-Khafaji RH, Aljunid SA, et al. Optimization of free space optics parameters: an optimum solution for bad weather conditions. Opt Int J Light Electron Opt 2013;124:3969-3973.

23. McArthur KB, Korevaar E. Comparison of laser beam propagation at $785 \mathrm{~nm}$ and $1550 \mathrm{~nm}$ in fog and haze for optical wireless communications III. Proc SPIE 2000;4214:26-37. 